CPP-94-37

DOE-ER-40757-060

UCD-95-4

February 1995

\title{
Hadronic Production of S-wave and P-wave Charmed Beauty Mesons via Heavy Quark Fragmentation
}

\author{
Kingman Cheung⿵ \\ Center for Particle Physics, University of Texas at Austin, Austin TX 78712 U.S.A. \\ Tzu Chiang Yuan \\ Davis Institute for High Energy Physics, Department of Physics, \\ University of California at Davis, Davis CA 95616 U.S.A.
}

\begin{abstract}
At hadron colliders the dominant production mechanism of $(\bar{b} c)$ mesons with large transverse momentum is due to parton fragmentation. We compute the rates and transverse momentum spectra for production of S-wave and $\mathrm{P}$-wave $(\bar{b} c)$ mesons at the Tevatron via the direct fragmentation of the bottom antiquark as well as the AltarelliParisi induced gluon fragmentation. Since all the radially and orbitally excited $(\bar{b} c)$ mesons below the $B D$ flavor threshold will cascade into the pseudoscalar ground state $B_{c}$ through electromagnetic and/or hadronic transitions, they all contribute to the inclusive production of $B_{c}$. The contributions of the excited S-wave and P-wave states to the inclusive production of $B_{c}$ are 58 and $23 \%$, respectively, and hence significant.
\end{abstract}

\footnotetext{
*Internet address: cheung@utpapa.ph.utexas.edu

†Internet address: yuantc@ucdhep.ucdavis.edu
} 


\section{Introduction}

Bound states composed of two heavy quarks such as $(c \bar{c}),(b \bar{b})$ and $(\bar{b} c)$ mesons, and $(c c q),(b b q)$ and $(b c q)$ baryons ( $q$ denotes a light quark) are of interests because they can be produced with sizable rates at the current high energy hadron or $e^{+} e^{-}$colliders. The Standard Model predictions of the production rates of these bound states can therefore be confronted with experimental data.

The direct production of heavy mesons like heavy quarkonia and $(\bar{b} c)$ bound states can provide very interesting tests for perturbative QCD. The production of $J / \psi$ and $\psi^{\prime}$ [1] at the Tevatron has already raised a lot of theoretical interests in explaining the excess of the experimental data above the lowest order perturbative QCD calculation [2], especially at the large transverse momentum $\left(p_{T}\right)$ region. The ideas of heavy quark and gluon fragmentation [3, $4,5,6,0,8,8$ have been successfully applied to explain the experimental data of the prompt $J / \psi$ production from CDF within a factor of five [9,10,11, 12]. Various attempts [13, 14, 15, 16] using the same fragmentation ideas have also been made to resolve the $\psi^{\prime}$ surplus problem observed at CDF.

Recently, the preliminary CDF results [17] also showed that the production rates of the $1 S, 2 S$, and $3 S \Upsilon$ states are in excess of the leading order calculation. While the $\Upsilon(1 S)$ and $\Upsilon(2 S)$ results can be partly explained by including the fragmentation contribution, the $\Upsilon(3 S)$ result showed an excess of about an order of magnitude over the QCD prediction even with the fragmentation contribution included [17]. One subtlety is that the relevant $p_{T}$ for the fragmentation contribution to dominate should be larger in the bottomonium system than in the charmonium system, such that fragmentation is only valid for $p_{T} \gtrsim(1-2) m_{b}$ in

the former case. Besides, one has to worry about the very small $p_{T}$ region because, unlike the charmonium system, the experimental triggering conditions on the muon pair coming from the bottomonium leptonic decay allow experimentalists to measure the transverse momentum of the bottomonium all the way down to about $0-1 \mathrm{GeV}$ [17]. In order to fully understand the $p_{T}$ spectrum of the bottomonium production, different production mechanisms have to 
be brought into picture to explain the production rates in different $p_{T}$ regions.

The $(\bar{b} c)$ meson system, which is intermediate between the $J / \psi$ and $\Upsilon$ families, is also an interesting physical system to study. The mass spectrum of the $(\bar{b} c)$ mesons can be predicted reliably from quarkonium potential models [18,19,20] without introducing any new parameters and their decay constants can be computed using QCD spectrum sum rules [19,20]. We will adopt the Particle Data Group [21] conventions, denoting the $1 S$ pseudoscalar $\left({ }^{1} S_{0}\right)$ and vector meson $\left({ }^{3} S_{1}\right)(\bar{b} c)$ states by $B_{c}$ and $B_{c}^{*}$, respectively. Higher radially and orbitally excited states are labeled by the standard spectroscopy notation: $n^{2 S+1} L_{J}$, where the integer $n$ is the principal quantum number, and $L, S$, and $J=L+S$ are respectively the orbital angular momentum, total spin, and total angular momentum of the bound state. In the $L S$ coupling scheme, for each principal quantum number $n$, the spin-singlet and the spin-triplet S-wave $(L=0)$ states are denoted by ${ }^{1} S_{0}$ and ${ }^{3} S_{1}$, respectively. Those for the P-wave $(L=1)$ and D-wave $(L=2)$ states are denoted by ${ }^{1} P_{1},{ }^{3} P_{J}(J=0,1,2)$, and ${ }^{1} D_{2}$, ${ }^{3} D_{J}(J=1,2,3)$, respectively.

According to the results of the potential model calculation [18], the first two sets $(n=1$ and $n=2)$ of S-wave states, the first $(n=1)$ and probably the entire second set $(n=2)$ of Pwave states, and the first set $(n=1)$ of D-wave states lie below the $B D$ flavor threshold. Since QCD interactions are diagonal in flavors, the annihilation channel of excited $(\bar{b} c)$ mesons can only occur through the weak gauge boson $(W)$ exchange and is therefore suppressed relative to the electromagnetic and hadronic transitions to other lower lying states. The excited states below the $B D$ threshold will cascade down into the ground state $B_{c}$ via emission of photons and/or pions, while the other states above the $B D$ threshold will decay rapidly into a pair of $B$ and $D$ mesons. Inclusive production of the $B_{c}$ meson therefore includes the production of the $n=1$ and $n=2 \mathrm{~S}$-wave and $\mathrm{P}$-wave states, and the $n=1 \mathrm{D}$-wave states.

The production of the S-wave $\left({ }^{1} S_{0}\right.$ and $\left.{ }^{3} S_{1}\right)$ states were first computed exactly to leading order in Ref. 22] at the $e^{+} e^{-}$machine, in particular at the $Z$ resonance. Later, it was realized [5:8] that the complicated formulas in these complete calculations can be simplified 
by a factorization approach. The dominant contribution in the leading order calculation can be factorized into a short distance piece, which describes the partonic process of the decay of $Z$ into a high energy $b \bar{b}$ pair, and a fragmentation piece describing how the $\bar{b}$ antiquark splits into the two S-wave states. The corresponding fragmentation functions $D_{\bar{b} \rightarrow B_{c}}(z)$ and $D_{\bar{b} \rightarrow B_{c}^{*}}(z)$, which are independent of the short-distance piece, were shown to be calculable by perturbative QCD at the heavy quark mass scale [5. Recently, the production of the S-wave states has also been computed at hadronic colliders like the Tevatron and the Large Hadron Collider (LHC) both by a complete $\mathcal{O}\left(\alpha_{s}^{4}\right)$ calculation [23, 24, 25, 26, 27] and by using the simpler fragmentation approach [28,29]. We note that unlike the $J / \psi$ production in hadronic collisions in which the major contributions come from (1) $g \rightarrow \chi_{c J}$ fragmentation followed by the decay $\chi_{c J} \rightarrow J / \psi+\gamma$ [9, 10,11,12], and (2) gluon fragmentation into a color-octet $(c \bar{c})^{3} S_{1}$ state which subsequently evolves nonperturbatively into $J / \psi$ [15, 16], the fragmentation diagrams for producing $(\bar{b} c)$ mesons form a subset of the whole set of $\mathcal{O}\left(\alpha_{s}^{4}\right)$ diagrams, which are the leading-order diagrams for producing $(\bar{b} c)$ mesons in hadronic collisions. It is therefore not clear that if the production of $(\bar{b} c)$ mesons in hadronic collisions is dominated by parton fragmentation. However, detailed calculations by Chang et al. [23], Slabospitsky [24], and Kolodziej et al [27] indicated that the fragmentation approach is valid for the S-wave production at the large transverse momentum region. We will discuss more about this later in the closing section.

In this paper, we study the hadronic production of $(\bar{b} c)$ mesons. We compute the production rates of the S-wave and $\mathrm{P}$-wave $(\bar{b} c)$ mesons at the Tevatron using the fragmentation approach. Intuitively, the dominant production mechanism of the $(\bar{b} c)$ mesons at the large transverse momentum region must arise from the direct fragmentation of the heavy $\bar{b}$ antiquark. The relevant question is whether experiments can probe the transverse momentum region where fragmentation dominates. Unfortunately, to answer this question it also requires a complete $\mathcal{O}\left(\alpha_{s}^{4}\right)$ calculation for the production of the $\mathrm{P}$-wave states, which is not available at the moment. Here we assume that the fragmentation approach also works for 
production of the $\mathrm{P}$-wave $(\bar{b} c)$ mesons in the transverse momentum range that is being probed experimentally at the Tevatron. In this work, we do not include the contributions from the D-wave states because the corresponding fragmentation functions are very small [30]. Although the production of the D-wave states is of great interest by themselves, they only contribute about $2 \%$ [30 to the inclusive production of $B_{c}$. In the near future, like other heavy quarkonia, the production of $(\bar{b} c)$ mesons at the Tevatron may therefore provide another interesting test for perturbative QCD. Although we will only show our results for the positively charged states $(\bar{b} c)$, all the results presented in this work also hold for the negatively charged states $(b \bar{c})$.

The organization of this paper is as follows. In the next section, we discuss in detail the general procedures to calculate the production cross sections using the fragmentation approach. In Sec. III we present the transverse momentum spectra and the integrated cross sections for the production of S-wave and P-wave $(\bar{b} c)$ states, as well as the inclusive production rate for the $B_{c}$ meson. Discussions and conclusions are made in Sec. IV. For completeness we also collect all the S-wave and P-wave fragmentation functions at the heavyquark mass scale in the Appendix.

Before leaving this preamble, we note that a preliminary result from CDF had provided a hint for the $B_{c}$ existence by looking at the production rate of $J / \psi+\pi$ in the mass bin of 6.1-6.4 GeV [31].

\section{Inclusive production cross sections in the fragmen- tation approach}

Theoretical calculations of production cross sections in high energy hadronic collisions are based on the idea of factorization. Factorization divides an inclusive or exclusive hadronic production process into short-distance pieces and long-distance pieces. The short-distance pieces are perturbatively calculable to any desired accuracy in QCD, while the long-distance 
pieces are in general not calculable within perturbation theory but can be parameterized as phenomenological functions, which can be determined by experiments. The factorization used here for the production of $(\bar{b} c)$ mesons divides the process into the production of a high energy parton (a $\bar{b}$ antiquark or a gluon) and the fragmentation of this parton into various $(\bar{b} c)$ states. The novel feature in our approach, which is due to a recent theoretical development [0, [], is that the relevant fragmentation functions at the heavy quark mass scale can be calculated in perturbative QCD to any desired accuracy. This is easily understood from the fact that the fragmentation of a $\bar{b}$ antiquark into a $(\bar{b} c)$ meson involves the creation of a $c \bar{c}$ pair out of the vacuum. The natural scale for this particular hadronization is of order of the mass of the quark pair being created. In our case, this scale is of order $m_{c}$, which is considerably larger than $\Lambda_{\mathrm{QCD}}$. One can therefore calculate reliably the fragmentation function as an series expansion in the strong coupling constant $\alpha_{s}$ using perturbative QCD.

The production of high energy partons also involves the factorization into the parton distribution functions inside the hadrons and the parton-parton hard scattering. Let $H$ denotes any $(\bar{b} c)$ meson states. The differential cross section $d \sigma / d p_{T}$ versus the transverse momentum $p_{T}$ of $H$ is given by

$$
\begin{aligned}
\frac{d \sigma}{d p_{T}}\left(p \bar{p} \rightarrow H\left(p_{T}\right) X\right)= & \sum_{i j} \int d x_{1} d x_{2} d z f_{i / p}\left(x_{1}, \mu\right) f_{j / \bar{p}}\left(x_{2}, \mu\right)\left[\frac{d \hat{\sigma}}{d p_{T}}\left(i j \rightarrow \bar{b}\left(p_{T} / z\right) X, \mu\right)\right. \\
& \left.\times D_{\bar{b} \rightarrow H}(z, \mu)+\frac{d \hat{\sigma}}{d p_{T}}\left(i j \rightarrow g\left(p_{T} / z\right) X, \mu\right) D_{g \rightarrow H}(z, \mu)\right] .
\end{aligned}
$$

The physical interpretation is as follows: a heavy $\bar{b}$ antiquark or a gluon is produced in a hard process with a transverse momentum $p_{T} / z$ and then it fragments into $H$ carrying a longitudinal momentum fraction $z$. We assume that $H$ is moving in the same direction as the fragmenting parton. In the above equation, $f_{i / p(\bar{p})}(x, \mu)$ 's are the parton distribution functions, $d \hat{\sigma}$ 's represent the subprocess cross sections, and $D_{i \rightarrow H}(z, \mu)$ 's are the parton fragmentation functions at the scale $\mu$. For production of the $\bar{b}$ antiquark, we include the subprocesses $g g \rightarrow b \bar{b}, g \bar{b} \rightarrow g \bar{b}$, and $q \bar{q} \rightarrow b \bar{b}$; while for the production of the gluon $g$, we include the subprocesses $g g \rightarrow g g, q \bar{q} \rightarrow g g$, and $g q(\bar{q}) \rightarrow g q(\bar{q})$. In Eq. (1), the factorization 
scale $\mu$ occurs in the parton distribution functions, the subprocess cross sections, and the fragmentation functions. In general, we can choose three different scales for these three entities. For simplicity and ease of estimating the uncertainties due to changes in scale, we choose a common scale $\mu$ for all three of them.

The physical production rates should be independent of choices of the scale $\mu$, because $\mu$ is just an artificial entity introduced to factorize the whole process into different parts in the renormalization procedure. However, this independence of scale can only be achieved if both the production of the high energy partons and the fragmentation functions are calculated to all orders in $\alpha_{s}$. So far, only the next-to-leading order $\hat{\sigma}$ 's and the leading order fragmentation functions are available, so the production cross sections do depend on the choice of $\mu$ to a certain degree. We will estimate the dependence on $\mu$ by varying the scale $\mu=(0.5-2) \mu_{R}$, where $\mu_{R}$ is our primary choice of scale

$$
\mu_{R}=\sqrt{p_{T}^{2}(\text { parton })+m_{b}^{2}}
$$

This choice of scale, which is of order $p_{T}$ (parton), avoids the large logarithms in the shortdistance part $\hat{\sigma}$ 's. However, we have to sum up the logarithms of order $\mu_{R} / m_{b}$ in the fragmentation functions. But this can be implemented by evolving the Altarelli-Parisi equations for the fragmentation functions.

\section{II.a Evolution of Fragmentation Functions}

The Altarelli-Parisi evolution equations for the fragmentation functions are

$$
\begin{aligned}
& \mu \frac{\partial}{\partial \mu} D_{\bar{b} \rightarrow H}(z, \mu)=\int_{z}^{1} \frac{d y}{y} P_{\bar{b} \rightarrow \bar{b}}(z / y, \mu) D_{\bar{b} \rightarrow H}(y, \mu)+\int_{z}^{1} \frac{d y}{y} P_{\bar{b} \rightarrow g}(z / y, \mu) D_{g \rightarrow H}(y, \mu), \\
& \mu \frac{\partial}{\partial \mu} D_{g \rightarrow H}(z, \mu)=\int_{z}^{1} \frac{d y}{y} P_{g \rightarrow \bar{b}}(z / y, \mu) D_{\bar{b} \rightarrow H}(y, \mu)+\int_{z}^{1} \frac{d y}{y} P_{g \rightarrow g}(z / y, \mu) D_{g \rightarrow H}(y, \mu),
\end{aligned}
$$

where $H$ denotes any $(\bar{b} c)$ states, and $P_{i \rightarrow j}$ are the usual Altarelli-Parisi splitting functions. The leading order expressions for $P_{i \rightarrow j}$ can be found in the Appendix. 
The boundary conditions for solving the above Altarelli-Parisi equations are the fragmentation functions $D_{\bar{b} \rightarrow H}\left(z, \mu_{0}\right)$ and $D_{g \rightarrow H}\left(z, \mu_{0}\right)$ that we can calculate by perturbative QCD at the initial scale $\mu_{0}$, which is of the order of the $b$-quark mass. At present, all the S-wave [5] and P-wave [7] fragmentation functions for $\bar{b} \rightarrow(\bar{b} c)$ have been calculated to leading order in $\alpha_{s}$. They are all collected in the Appendix. The initial scale $\mu_{0}$ for $D_{\bar{b} \rightarrow H}\left(z, \mu_{0}\right)$ is chosen to be $\mu_{0}=m_{b}+2 m_{c}$, which is the minimum virtuality of the fragmenting $\bar{b}$ antiquark [5]. On the other hand, since the initial gluon fragmentation function $D_{g \rightarrow H}\left(z, \mu_{0}\right)$ is suppressed by one extra power of $\alpha_{s}$ relative to $D_{\bar{b} \rightarrow H}\left(z, \mu_{0}\right)$, we simply choose the initial gluon fragmentation function to be $D_{g \rightarrow H}\left(z, \mu_{0}\right)=0$ for $\mu_{0} \leq 2\left(m_{b}+m_{c}\right)$ - the minimum virtuality of the fragmenting gluon [29]. We can also examine the relative importance of these fragmentation functions. The initial $D_{\bar{b} \rightarrow H}\left(z, \mu_{0}\right)$ is of order $\alpha_{s}^{2}$, whereas the initial $D_{g \rightarrow H}\left(z, \mu_{0}\right)$ is of order $\alpha_{s}^{3}$ and has been set to be zero for $\mu_{0} \leq 2\left(m_{b}+m_{c}\right)$ as discussed above. But when the scale is evolved up to a higher scale $\mu, D_{\bar{b} \rightarrow H}(z, \mu)$ is still of order $\alpha_{s}^{2}$, while the induced $D_{g \rightarrow H}(z, \mu)$ is of order $\alpha_{s}^{3} \log \left(\mu / \mu_{0}\right)$. At a sufficiently large scale $\mu$ the logarithmic enhancement can offset the extra suppression factor of $\alpha_{s}$. Thus the Altarelli-Parisi induced gluon fragmentation functions can be as important as the $\bar{b}$ antiquark fragmentation, even though the initial gluon fragmentation functions are suppressed. While these Altarelli-Parisi induced gluon fragmentation functions play only a moderate role at the Tevatron, they will play a more significant role at the LHC [29].

To obtain the fragmentation functions at an arbitrary scale greater than $\mu_{0}$, we numerically integrate the Altarelli-Parisi evolution equations (3)-(国) with the boundary conditions described in the above paragraph. Since the initial light-quark fragmentation functions $D_{q \rightarrow H}\left(z, \mu_{0}\right)$ are of order $\alpha_{s}^{4}$, one can set them to be zero as well for $\mu_{0} \leq 2\left(m_{b}+m_{c}\right)$. One may ask if the light-quark fragmentation functions can be induced in the same manner as in the gluon case by Altarelli-Parisi evolution. Since both the Altarelli-Parisi splitting functions $P_{q \rightarrow q}$ and $P_{q \rightarrow g}$ are total plus-functions, the induced light-quark fragmentation functions $D_{q \rightarrow H}(z)$ can only be total plus-functions or vanishing identically. We do not an- 
ticipate that these induced light-quark fragmentation functions, if nontrivial, will play any significant role in our analysis.

\section{Numerical results}

Leading order QCD formulas are employed for the parton-level scattering cross sections and CTEQ(2M) [32] is used for the initial parton distributions. The inputs to the initial fragmentation functions are the heavy quark masses $m_{b}$ and $m_{c}$, and the nonperturbative parameters associated with the wavefunctions of the bound states. For the S-wave states there is only one nonperturbative parameter, which is the radial wavefunction $R_{n S}(0)$ at the origin. However for the P-wave fragmentation functions we have two nonperturbative parameters $H_{1}$ and $H_{8}^{\prime}$ associated with the color-singlet and the color-octet mechanisms, respectively [33]. Two of the $\mathrm{P}$-wave states $\left({ }^{1} P_{1}\right.$ and $\left.{ }^{3} P_{1}\right)$ are mixed to form the two physical states, denoted by $\left|1^{+}\right\rangle$and $\left|1^{+\prime}\right\rangle$. Further details of the mixings can be found in Refs. [7, 18].

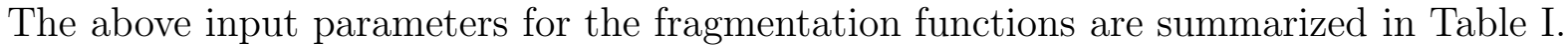

As mentioned in the previous section, we have set the scale $\mu$ in the parton distribution functions, subprocess cross sections, and the fragmentation functions to be the same. We will later vary $\mu$ between $0.5 \mu_{R}$ and $2 \mu_{R}$, where $\mu_{R}$ is given in Eq. (2), to study the dependence

on the choice of scale. For the strong coupling constant $\alpha_{s}(\mu)$ entered in the subprocess cross sections, we employ the following simple expression

$$
\alpha_{s}(\mu)=\frac{\alpha_{s}\left(M_{Z}\right)}{1+\frac{33-2 n_{f}}{6 \pi} \alpha_{s}\left(M_{Z}\right) \ln \left(\frac{\mu}{M_{Z}}\right)},
$$

where $n_{f}$ is the number of active flavors at the scale $\mu$ and $\alpha_{s}\left(M_{Z}\right)=0.118$. In order to simulate the detector coverage at the Tevatron, we impose the following acceptance cuts on the transverse momentum and rapidity of the $(\bar{b} c)$ state $H$ :

$$
p_{T}(H)>6 \mathrm{GeV} \quad \text { and } \quad|y(H)|<1 .
$$

The numerical results of the $p_{T}$ spectra for the $(\bar{b} c)$ state $H$ with various spin-orbital 
quantum numbers are shown in Fig. 1 1 and Fig. 2 for the cases of principal quantum number $n=1$ and $n=2$, respectively. The integrated cross sections versus $p_{T}^{\min }(H)$ are also shown in Figs. 3 and 4 for the cases of $n=1$ and $n=2$, respectively.

Now we can predict the inclusive production rate of the $B_{c}$ meson. As the annihilation channel is suppressed relative to the electromagnetic/hadronic transitions, all the $(\bar{b} c)$ excited states below the $B D$ threshold will decay eventually into the ground state $B_{c}$ via emission of photons or pions. Since the energies of these emitted photons and/or pions are limited by the small mass differences between the initial and final $(\bar{b} c)$ states, the transverse momenta of the $(\bar{b} c)$ mesons will not be altered appreciably during the cascades. Therefore, we can simply add up the $p_{T}$ spectra (Fig. 目and Fig. 2) of all the states to represent the $p_{T}$ spectrum of the inclusive $B_{c}$ production. Similarly, we can add up the integrated cross sections (Fig. 通 and Fig. (1) to represent the integrated cross section for the inclusive $B_{c}$ production. Thus, we can obtain the inclusive production rate of $B_{c}$ as a function of $p_{T}^{\min }\left(B_{c}\right)$. Table $\mathbb{\prod}$ gives the inclusive cross sections for the $B_{c}$ meson at the Tevatron as a function of $p_{T}^{\min }\left(B_{c}\right)$, including all the contributions from the $n=1$ and $n=2 \mathrm{~S}$-wave and P-wave states. These cross sections should almost represent the inclusive production of $B_{c}$ by fragmentation, because the contributions from the D-wave states are expected to be minuscule. The results for $\mu=\mu_{R} / 2, \mu_{R}$, and $2 \mu_{R}$ are also shown. The variation of the integrated cross sections with the scale $\mu$ is always within a factor of two, and only about $20 \%$ for $p_{T}>10 \mathrm{GeV}$. We will discuss more about the dependence of scale in the next section.

At the end of Run Ib at the Tevatron, the total accumulated luminosities can be up to $100-150 \mathrm{pb}^{-1}$ or more. With $p_{T}>6 \mathrm{GeV}$, there are about $5 \times 10^{5} B_{c}^{+}$mesons. The lifetime of the $B_{c}$ meson has been estimated to be of order 1-2 picosecond [18], which is long enough to leave a displaced vertex in a silicon vertex detector. Besides, $B_{c}$ decays into $J / \psi+X$ very often, where $X$ can be a $\pi^{+}, \rho^{+}$, or $\ell^{+} \nu_{l}$, and $J / \psi$ can be detected easily through its leptonic decay modes. The inclusive branching ratio of $B_{c} \rightarrow J / \psi+X$ is about $10 \%$ [34]. When $X$ is $e^{+} \nu_{e}$ or $\mu^{+} \nu_{\mu}$, we will obtain the striking signature of three-charged 
leptons coming off from a common secondary vertex. The combined branching ratio of $B_{c} \rightarrow J / \psi \ell^{+} \nu_{\ell} \rightarrow \ell^{\prime+} \ell^{\prime-} \ell^{+} \nu_{\ell}\left(\ell, \ell^{\prime}=e, \mu\right)$ is about $0.2 \%$. This implies that there will be of order $10^{3}$ such distinct events for $100 \mathrm{pb}^{-1}$ luminosity at the Tevatron. Even taking into account the imperfect detection efficiencies, there should be enough events for confirmation. However, this mode does not afford the full reconstruction of the $B_{c}$. If $X$ is some hadronic states, e.g., pions, the events can be fully reconstructed and the $B_{c}$ meson mass can be measured. The process $B_{c} \rightarrow J / \psi+\pi^{+} \rightarrow \ell^{+} \ell^{-} \pi^{+}$is likely to be the discovery mode for $B_{c}$. Its combined branching ratio is about $0.03 \%$, which implies about 300 such distinct events at the Tevatron with a luminosity of $100 \mathrm{pb}^{-1}$.

After the next fixed target runs at the Tevatron, the Main Injector will be installed in 1996-1997 according to the present plan [35]. The Main Injector will give a significant boost in the luminosity while the center-of-mass energy stays the same. The upgraded luminosity is estimated to be about ten times larger than its present value. This enables Run II to accumulate a total luminosity of $1-2 \mathrm{fb}^{-1}$, which implies that about $10^{7}-10^{8} B_{c}$ mesons will be produced. With the Main Injector installed, it might be possible to produce the D-wave $(\bar{b} c)$ states with sizable rates.

Tevatron will continue running until the next generation of hadronic colliders, e.g., the LHC. The present design of the LHC is at a center-of-mass energy of $14 \mathrm{TeV}$ and the yearly luminosity is of order $100 \mathrm{fb}^{-1}$. In Table [II], we show the inclusive cross sections for the $B_{c}$ meson at the LHC as a function of $p_{T}^{\min }\left(B_{c}\right)$ including the contributions from $n=1$ and $n=2$ S-wave and P-wave states. With the assumed $100 \mathrm{fb}^{-1}$ luminosity there are about $3 \times 10^{9}$ $B_{c}$ mesons with $p_{T}>10 \mathrm{GeV}$ and $\left|y\left(B_{c}\right)\right|<2.5$. With such a high luminosity at LHC, one expects sizable numbers of the various D-wave $(\bar{b} c)$ states to be produced as well. The LHC will then be a copious source of $(\bar{b} c)$ mesons such that their properties e.g., spectroscopy and decays, can be thoroughly studied. In addition, the mixing and $\mathrm{CP}$ violation studies are possible. For example, one can use $B_{c}$ to tag the flavor of $B_{s}$ in the decay $B_{c}^{+} \rightarrow B_{s}^{0} \ell^{+} \nu_{l}$ for the studies of $B_{s}^{0}-\bar{B}_{s}^{0}$ mixing. Also, $\mathrm{CP}$ violations in the $B_{c}$ system can be studied by 
looking at the difference in the partial decay widths of $B_{c}^{+} \rightarrow X$ and $B_{c}^{-} \rightarrow \bar{X}$.

\section{Discussions and conclusions}

First, we briefly discuss the various sources of uncertainties in our calculation. One uncertainty comes from the use of the naive Altarelli-Parisi evolution equations. As pointed out in Refs. [9, [36], the naive Altarelli-Parisi equations in Eqs. (3)-(41) do not respect the phase space constraints. Inhomogeneous evolution equations were then advocated to remedy for these problems. The major effect is to correct the unphysical blow-up of the evolved gluon fragmentation functions when $z$ gets too close to 0 . The corrected gluon fragmentation functions, instead of having the unphysical blow-up at $z=0$, turn to zero smoothly below a certain threshold value of $z$. Despite the dramatic changes of the evolved gluon fragmentation functions for small $z$ values, this effect will not show up easily in our calculation because the small $z$ region is very likely excluded by the transverse momentum cut imposed on the $(\bar{b} c)$ mesons. Therefore, in this paper we keep on using the homogeneous Altarelli-Parisi equations in Eqs. (8)-(4) to evolve our fragmentation functions.

A second source of uncertainty is due to the choice of the factorization scale $\mu$. We show in Fig. 5 the dependence of the differential cross sections on the factorization scale $\mu$ by plotting the results for the various choices of $\mu=\mu_{R} / 2, \mu_{R}$, and $2 \mu_{R}$. For clarity we only show the curves for the $1{ }^{1} S_{0}$ and $1{ }^{3} P_{0}$ states in Fig. 5. The behaviors for other states are similar. Note that in the $\mu=\mu_{R} / 2, \mu_{R}$, and $2 \mu_{R}$ curves, the running scales used in the strong coupling constant $\alpha_{s}$, which entered in $d \hat{\sigma}$ 's and in the parton distribution functions $f_{i}(x)$ 's are equal to $\mu$, while the running scale used in the fragmentation functions is set to be max $\left(\mu, \mu_{0}\right)$, where $\mu_{0}$ is the prescribed initial scale for the fragmentation functions. Figure 5 shows that the change in the factorization scale $\mu$ gives different results for the differential cross sections. The $\mu=2 \mu_{R}$ curves show that the differential cross sections increase (decrease)

only slightly at the low (high) $p_{T}$ region. Although the $\mu=\mu_{R} / 2$ curves demonstrate 
larger changes in the differential cross sections, the variations are always within a factor of two. The integrated cross sections at various $p_{T}^{\min }\left(B_{c}\right)$, as already shown in Table [1], also indicate lesser sensitivity in the scale as one increases $p_{T}^{\min }\left(B_{c}\right)$. The variations of differential cross sections with the scale $\mu$ demonstrate the effects of the next-to-leading order (NLO) corrections. Only if all the NLO corrections are calculated for each piece contained in Eq. (11), namely the parton distribution functions, the parton-parton hard scattering cross sections, and the fragmentation functions, can these variations be reduced substantially. Since the perturbative QCD fragmentation functions are only calculated to leading order, the NLO calculations of the perturbative QCD fragmentation functions can provide an improvement to our calculation. However, due to the rather weak dependence on the scale in our leading order calculation, our results should be rather stable under higher order perturbative corrections.

Other uncertainties come from the input parameters to the boundary conditions of the fragmentation functions. These are the heavy quark masses $m_{b}$ and $m_{c}$, and the nonperturbative parameters describing the bound states. Slight changes in $m_{c}$ and $m_{b}$ could possibly lead to appreciable changes in the fragmentation functions, as indicated by the $m^{3}$ and $m^{5}$ dependence, respectively in the denominators of the S-wave and P-wave fragmentation functions. (Note that $H_{1} / m \approx 9\left|R^{\prime}(0)\right|^{2} /\left(32 \pi m^{5}\right)$.) However, in the numerators the wavefunctions at the origin $|R(0)|^{2}$ and $\left|R^{\prime}(0)\right|^{2}$ also scale like $m^{3}$ and $m^{5}$, respectively. Therefore the dependence on the heavy quark masses should be mild in the fragmentation functions. The color-octet parameters $H_{8}^{\prime}$ 's are associated with the additional color-octet contributions and they are not well determined. However, we do not expect that they will play any significant role in the present context and only refer our readers to Ref.[0] where some discussions of these parameters can be found.

There was a controversy in the recent literature [23, 24, 25, 26, 27] concerning about the importance of parton fragmentation in the production of the $B_{c}\left(B_{c}^{*}\right)$ meson at hadronic colliders. The controversy arises because the Feynman diagrams responsible for the $\bar{b}$ antiquark fragmentation form a subset of the whole set of Feynman diagrams, which contribute 
at the order of $\alpha_{s}^{4}$. Thus, there is a competition between the fragmentation contribution and the non-fragmentation contribution. Some authors had referred to the latter contribution as recombination. So far, five independent groups [23, 24, 25, 26, 27, have presented such a complete $\mathcal{O}\left(\alpha_{s}^{4}\right)$ calculation. Chang et al. [23] and Slabospitsky [24] agreed that the fragmentation contribution dominates at the large transverse momentum region. However, we could not find in their work the precise value of $p_{T}$ at which the fragmentation contribution begins to dominate. On the other hand, Berezhnoy et al. 25. claimed that fragmentation never dominates for all kinematical region of $p_{T}$ and the recombination diagrams can never be ignored. Independently, Masetti and Sartogo [26] have recently obtained results claimed to be consistent with Berezhnoy et al [25]. There are certainly discrepancies among these calculations. Most recently, Kolodziej et al [27] performed yet another independent calculations using two different methods - the usual trace and the modern helicity amplitude techniques. Both methods agreed with each other numerically to very high accuracy, according to the authors. Kolodziej et al. [27] also did a very thorough comparison with previous exact calculations by comparing numerically their matrix elements with other groups [23,24,25], but could not find agreement using identical input parameters. f Furthermore, Kolodziej et al. [27] did a comparison of the production of $B_{c}$ mesons between the exact calculation and the fragmentation approximation, and found that the fragmentation approach is indeed valid for $p_{T} \geq 10 \mathrm{GeV}$ ! Because of the conclusions of Ref. [27], we believe that the fragmentation contribution should begin to dominate the production of $(\bar{b} c)$ mesons at $p_{T} \gtrsim 10 \mathrm{GeV}$. Also, we would like to emphasize that the detector coverage and performance do require a minimum transverse momentum cut and a rapidity cut on the $B_{c}$ meson. Thus, the non-fragmentation (recombination) contribution, which is substantial at low $p_{T}$, becomes less important as the lowest $p_{T}$ range is being excluded.

\footnotetext{
${ }^{\ddagger}$ In their most recently revised versions, both groups of Chang et al. (the second entry of Ref. [23]) and Berezhnoy et al. 25 confirmed the results of Kolodziej et al. [27]. Thanks to an anonymous referee for pointing out the situation.
} 
The main result of this paper is the inclusive cross section of the ground state $B_{c}$ at the Tevatron. We have included in this work all the contributions from the direct $\bar{b}$ fragmentation and induced gluon fragmentation, as well as the cascade contributions from all the excited $\mathrm{S}$-wave and $\mathrm{P}$-wave states below the $B D$ threshold. Previous result presented by one of us in Ref. 28] only included the direct $\bar{b}$ fragmentation into S-wave states, since the Pwave fragmentation functions were not available at that time. Later it was pointed out in Ref. [29] that the induced gluon fragmentation contribution is nonnegligible. The induced gluon fragmentation contribution is about $20-30 \%(30-40 \%)$ of the direct $\bar{b}$ fragmentation contribution at the Tevatron (LHC) energies. The two sets of P-wave states included in this work contribute at the level of $30 \%$. Furthermore, we have employed here a more updated set of parton distribution functions than those used in Refs. [28,29]. As a result, the cross sections increase roughly by $25 \%$, mainly due to the increase in the gluon luminosities. When all these aforementioned differences are taken into account, the net result is about $70-80 \%$ (a factor of two) larger than the previous result presented in Ref.[28] at the Tevatron (LHC).

To recap, in this paper we have performed calculations of the transverse momentum spectra and integrated cross sections for the S- and P-wave $(\bar{b} c)$ mesons. We have also predicted the inclusive production rate for the $B_{c}$ meson, and found that there should be enough signature events to confirm the existence of $B_{c}$ at the Tevatron, whereas the LHC will be a copious source of $B_{c}$. Nevertheless, one should keep in mind that what we have calculated in this paper represents only the contribution from fragmentation, while there should also be other non-fragmentation contributions, especially at the low $p_{T}$ region $\left(p_{T} \lesssim\right.$ $10 \mathrm{GeV}$ ). At least, our results represent a lower bound of the production rate for $B_{c}$. In the near future, the production of $B_{c}$ at the Tevatron would provide another interesting test for perturbative QCD, and we expect a very exciting experimental program of $B_{c}$ at the LHC. 


\section{Acknowledgement}

This work was supported in part by the United States Department of Energy under Grant Numbers DE-FG03-93ER40757 and DE-FG03-91ER40674. 


\section{Appendix}

The Altarelli-Parisi evolution equations were given in Eqs. (3)-(4). For convenience, we also list all the Altarelli-Parisi kernels in the following,

$$
\begin{aligned}
P_{\bar{b} \rightarrow \bar{b}}(x, \mu) & =\frac{4 \alpha_{s}(\mu)}{3 \pi}\left(\frac{1+x^{2}}{1-x}\right)_{+}, \\
P_{b \rightarrow g}(x, \mu) & =\frac{4 \alpha_{s}(\mu)}{3 \pi}\left(\frac{1+(1-x)^{2}}{x}\right)_{+}, \\
P_{g \rightarrow \bar{b}}(x, \mu) & =\frac{\alpha_{s}(\mu)}{2 \pi}\left(x^{2}+(1-x)^{2}\right) \\
P_{g \rightarrow g}(x, \mu) & =\frac{6 \alpha_{s}(\mu)}{\pi}\left(\frac{x}{(1-x)_{+}}+\frac{1-x}{x}+x(1-x)+\left(\frac{11}{12}-\frac{n_{f}}{18}\right) \delta(1-x)\right),
\end{aligned}
$$

where $n_{f}$ is the number of active flavors below the scale $\mu$.

The fragmentation functions $D_{\bar{b} \rightarrow H}\left(z, \mu_{0}\right)$ for the $\mathrm{S}$-wave and P-wave $(\bar{b} c)$ states have been obtained to leading order in strong coupling constant $\alpha_{s}$ at the heavy quark mass scale $\mu_{0}=m_{b}+2 m_{c}$. We introduce the following notations: $r=m_{c} /\left(m_{b}+m_{c}\right), \bar{r}=1-r$, and $m=m_{b} m_{c} /\left(m_{b}+m_{c}\right)$. The nonperturbative parameters are $R_{n S}(0)$ for the S-wave states, and $H_{1}(n)$ and $H_{8}^{\prime}(n)$ for the $\mathrm{P}$-wave states. The mixing angle between the ${ }^{1} P_{1}$ and ${ }^{3} P_{1}$ states is denoted by $\cos \theta_{\text {mix }}$. The numerical values of these input parameters can be inferred from potential model calculations (see for example [18]) and are listed in Table $[$ for convenience. The scale of the strong coupling constant entered in $D_{\bar{b} \rightarrow H}\left(z, \mu_{0}\right)$ is set to be $2 m_{c}$.

The S-wave fragmentation functions are [5]

$$
\begin{aligned}
D_{\bar{b} \rightarrow \bar{b} c\left(n^{1} S_{0}\right)}\left(z, \mu_{0}\right)= & \frac{2 \alpha_{s}\left(2 m_{c}\right)^{2}\left|R_{n S}(0)\right|^{2}}{81 \pi m_{c}^{3}} \frac{r z(1-z)^{2}}{(1-\bar{r} z)^{6}} \\
\times & {\left[6-18(1-2 r) z+\left(21-74 r+68 r^{2}\right) z^{2}\right.} \\
& \left.-2 \bar{r}\left(6-19 r+18 r^{2}\right) z^{3}+3 \bar{r}^{2}\left(1-2 r+2 r^{2}\right) z^{4}\right],
\end{aligned}
$$




$$
\begin{aligned}
D_{\bar{b} \rightarrow \bar{b} c\left(n^{3} S_{1}\right)}\left(z, \mu_{0}\right)= & \frac{2 \alpha_{s}\left(2 m_{c}\right)^{2}\left|R_{n S}(0)\right|^{2}}{27 \pi m_{c}^{3}} \frac{r z(1-z)^{2}}{(1-\bar{r} z)^{6}} \\
\times & {\left[2-2(3-2 r) z+3\left(3-2 r+4 r^{2}\right) z^{2}-2 \bar{r}\left(4-r+2 r^{2}\right) z^{3}\right.} \\
& \left.+\bar{r}^{2}\left(3-2 r+2 r^{2}\right) z^{4}\right]
\end{aligned}
$$

Polarized S-wave fragmentation functions can also be found in Ref. [37 but they are not used in this work.

The P-wave fragmentation functions consist of two terms, the color-singlet piece $\left(H_{1}\right.$ term) and the color octet piece $\left(H_{8}^{\prime}\right.$ term $)$ [7]:

$$
\begin{aligned}
D_{\bar{b} \rightarrow \bar{b} c\left(n^{1} P_{1}\right)}\left(z, \mu_{0}\right) & =\frac{H_{1(\bar{b} c)}(n)}{m} D_{\bar{b} \rightarrow \bar{b} c\left({ }^{1} P_{1}\right)}^{(1)}(z, \Lambda)+3 \frac{H_{8(\bar{b} c)}^{\prime}(\Lambda)}{m} D_{\bar{b} \rightarrow \bar{b} c\left({ }^{1} S_{0}\right)}^{(8)}(z) \\
D_{\bar{b} \rightarrow \bar{b} c\left(n^{3} P_{J}\right)}\left(z, \mu_{0}\right) & =\frac{H_{1(\bar{b} c)}(n)}{m} D_{\bar{b} \rightarrow \bar{b} c\left({ }^{3} P_{J}\right)}^{(1)}(z, \Lambda)+(2 J+1) \frac{H_{8(\bar{b} c)}^{\prime}(\Lambda)}{m} D_{\bar{b} \rightarrow \bar{b} c\left({ }^{3} S_{1}\right)}^{(8)}(z)
\end{aligned}
$$

where $\Lambda$ is a factorization scale for the $\mathrm{P}$-wave fragmentation functions. It separates the reduced heavy quark mass scale $m$ and the scale $m v$ which characterizes the bound state structures with $v$ being the typical relative velocity of the heavy quark and antiquark inside the bound state. In our numerical work, we will choose $\Lambda \sim m$.

At leading order the color-singlet pieces do not depend on $\Lambda$ and they are given by

$$
\begin{aligned}
D_{\bar{b} \rightarrow \bar{b} c\left({ }^{1} P_{1}\right)}^{(1)}(z) & =\frac{16 \alpha_{s}^{2}\left(2 m_{c}\right)}{243} \frac{r \bar{r}^{3} z(1-z)^{2}}{(1-\bar{r} z)^{8}}\left[6-6\left(4 r^{2}-8 r+5\right) z\right. \\
& +\left(32 r^{4}-96 r^{3}+250 r^{2}-210 r+69\right) z^{2} \\
& +8 \bar{r}\left(4 r^{4}+12 r^{3}-48 r^{2}+37 r-12\right) z^{3} \\
& +2 \bar{r}^{2}\left(16 r^{4}+161 r^{2}-114 r+42\right) z^{4}-6 \bar{r}^{3}\left(4 r^{3}+28 r^{2}-15 r+7\right) z^{5} \\
& \left.+\bar{r}^{4}\left(46 r^{2}-14 r+9\right) z^{6}\right],
\end{aligned}
$$




$$
\begin{aligned}
D_{\bar{b} \rightarrow \bar{b} c\left({ }^{3} P_{0}\right)}^{(1)}(z) & =\frac{16 \alpha_{s}^{2}\left(2 m_{c}\right)}{729} \frac{r \bar{r}^{3} z(1-z)^{2}}{(1-\bar{r} z)^{8}}\left[6(4 r-1)^{2}+6(4 r-1)\left(20 r^{2}-16 r+5\right) z\right. \\
& +\left(832 r^{4}-1456 r^{3}+1058 r^{2}-362 r+63\right) z^{2} \\
& -8 \bar{r}\left(100 r^{4}-184 r^{3}+118 r^{2}-22 r+9\right) z^{3} \\
& +2 \bar{r}^{2}\left(416 r^{4}-776 r^{3}+369 r^{2}+42 r+24\right) z^{4} \\
& -2 \bar{r}^{3}\left(240 r^{4}-516 r^{3}+232 r^{2}+59 r+9\right) z^{5} \\
& \left.+\bar{r}^{4}\left(96 r^{4}-240 r^{3}+134 r^{2}+34 r+3\right) z^{6}\right], \\
& \\
& =\frac{32 \alpha_{s}^{2}\left(2 m_{c}\right)}{243} \frac{r \bar{r}^{3} z(1-z)^{2}}{(1-\bar{r} z)^{8}}[6+6(4 r-5) z \\
& +\left(16 r^{4}+64 r^{2}-98 r+63\right) z^{2}+8 \bar{r}\left(2 r^{4}+2 r^{3}-13 r^{2}+11 r-9\right) z^{3} \\
D_{\bar{b} \rightarrow \bar{b} c\left({ }^{3} P_{1}\right)}^{(1)}(z) & 2 \bar{r}^{2}\left(8 r^{4}-16 r^{3}+47 r^{2}-18 r+24\right) z^{4}+2 \bar{r}^{3}\left(8 r^{3}-24 r^{2}+r-9\right) z^{5} \\
+ & \left.\bar{r}^{4}\left(12 r^{2}+2 r+3\right) z^{6}\right],
\end{aligned}
$$

and

$$
\begin{aligned}
D_{\bar{b} \rightarrow \bar{b} c\left({ }^{3} P_{2}\right)}^{(1)}(z) & =\frac{64 \alpha_{s}^{2}\left(2 m_{c}\right)}{729} \frac{r \bar{r}^{5} z(1-z)^{2}}{(1-\bar{r} z)^{8}}[12+12(2 r-5) z \\
& +\left(92 r^{2}-76 r+135\right) z^{2}+4\left(10 r^{3}-54 r^{2}+31 r-45\right) z^{3} \\
& +2\left(46 r^{4}-16 r^{3}+123 r^{2}-78 r+75\right) z^{4} \\
& -4 \bar{r}\left(6 r^{4}+9 r^{3}+40 r^{2}-13 r+18\right) z^{5} \\
& \left.+\bar{r}^{2}\left(12 r^{4}-12 r^{3}+55 r^{2}-10 r+15\right) z^{6}\right]
\end{aligned}
$$

The ${ }^{1} P_{1}$ and ${ }^{3} P_{1}$ states are mixed in general, giving rise to the following physical mass eigenstates $\left|1^{+^{\prime}}\right\rangle$ and $\left|1^{+}\right\rangle$,

$$
\begin{aligned}
\left|1^{+^{\prime}}\right\rangle & =\cos \theta_{\text {mix }}\left|{ }^{1} P_{1}\right\rangle-\sin \theta_{\text {mix }}\left|{ }^{3} P_{1}\right\rangle \\
\left|1^{+}\right\rangle & =\sin \theta_{\text {mix }}\left|{ }^{1} P_{1}\right\rangle+\cos \theta_{\text {mix }}\left|{ }^{3} P_{1}\right\rangle
\end{aligned}
$$


Thus, in general, we have

$$
\begin{aligned}
D_{\bar{b} \rightarrow \bar{b} c\left(1^{\prime}\right)}^{(1)}(z)= & \cos ^{2} \theta_{\text {mix }} D_{\bar{b} \rightarrow \bar{b} c\left({ }^{1} P_{1}\right)}^{(1)}(z)+\sin ^{2} \theta_{\text {mix }} D_{\bar{b} \rightarrow \bar{b} c\left({ }^{3} P_{1}\right)}^{(1)}(z) \\
& -\sin \theta_{\text {mix }} \cos \theta_{\text {mix }} D_{\text {mix }}^{(1)}(z), \\
D_{\bar{b} \rightarrow \bar{b} c\left(1^{+}\right)}^{(1)}(z)= & \sin ^{2} \theta_{\text {mix }} D_{\bar{b} \rightarrow \bar{b} c\left({ }^{1} P_{1}\right)}^{(1)}(z)+\cos ^{2} \theta_{\text {mix }} D_{\bar{b} \rightarrow \bar{b} c\left({ }^{3} P_{1}\right)}^{(1)}(z) \\
& +\sin \theta_{\text {mix }} \cos \theta_{\text {mix }} D_{\text {mix }}^{(1)}(z),
\end{aligned}
$$

with

$$
\begin{aligned}
D_{\text {mix }}^{(1)}(z) & =-\frac{32 \sqrt{2} \alpha_{s}^{2}\left(2 m_{c}\right)}{243} \frac{r \bar{r}^{3} z(1-z)^{2}}{(1-\bar{r} z)^{6}}\left[6-6\left(2 r^{2}-4 r+3\right) z\right. \\
& +\left(24 r^{3}+52 r^{2}-52 r+21\right) z^{2}+2 \bar{r}\left(14 r^{3}-6 r^{2}+15 r-6\right) z^{3} \\
& \left.-\bar{r}^{2}\left(2 r^{2}+8 r-3\right) z^{4}\right] .
\end{aligned}
$$

Finally, the color-octet pieces are given by

$$
\begin{gathered}
D_{\bar{b} \rightarrow \bar{b} c\left({ }^{1} S_{0}\right)}^{(8)}(z)=\frac{\alpha_{s}^{2}\left(2 m_{c}\right)}{162} \frac{r \bar{r}^{3} z(1-z)^{2}}{(1-\bar{r} z)^{6}}\left[6-18(1-2 r) z+\left(21-74 r+68 r^{2}\right) z^{2}\right. \\
\left.-2 \bar{r}\left(6-19 r+18 r^{2}\right) z^{3}+3 \bar{r}^{2}\left(1-2 r+2 r^{2}\right) z^{4}\right]
\end{gathered}
$$

and

$$
\begin{gathered}
D_{\bar{b} \rightarrow \bar{b} c\left({ }^{(3} S_{1}\right)}^{(8)}(z)=\frac{\alpha_{s}^{2}\left(2 m_{c}\right)}{162} \frac{r \bar{r}^{3} z(1-z)^{2}}{(1-\bar{r} z)^{6}}\left[2-2(3-2 r) z+3\left(3-2 r+4 r^{2}\right) z^{2}\right. \\
\left.-2 \bar{r}\left(4-r+2 r^{2}\right) z^{3}+\bar{r}^{2}\left(3-2 r+2 r^{2}\right) z^{4}\right] .
\end{gathered}
$$




\section{References}

[1] F. Abe et al. (CDF Coll.), Phys. Rev. Lett. 69, 3704 (1992).

[2] E. W. N. Glover, A. D. Martin, and W. J. Stirling, Z. Phys. C38, 473 (1988).

[3] E. Braaten and T. C. Yuan, Phys. Rev. Lett. 71, 1673 (1993).

[4] E. Braaten, K. Cheung, and T. C. Yuan, Phys. Rev. D48, 4230 (1993).

[5] E. Braaten, K. Cheung, and T. C. Yuan, Phys. Rev. D48, R5049 (1993).

[6] E. Braaten and T. C. Yuan, Phys. Rev. D50, 3176 (1994).

[7] T. C. Yuan, Phys. Rev. D50, 5664 (1994).

[8] C.-H. Chang and Y.-Q. Chen, Phys. Lett. B284, 127 (1992); Phys. Rev. D46, 3845 (1992), D50, 6013 (1994); Y.-Q. Chen, Phys. Rev. D48, 5158 (1993), D50, 6013 (1994).

[9] E. Braaten, M. Doncheski, S. Fleming, and M. Mangano, Phys. Lett. B333, 548 (1994).

[10] M. Cacciari and M. Greco, Phys. Rev. Lett. 73, 1586 (1994).

[11] D. P. Roy and K. Sridhar, Phys. Lett. B339, 141 (1994).

[12] P. Cho, M. Wise, and S. Trivedi, Phys. Rev. D51, 2039 (1995).

[13] F. E. Close, Phys. Lett. B342, 369 (1995).

[14] P. Cho and M. Wise, Phys. Lett. B346, 129 (1995).

[15] E. Braaten and S. Fleming, Phys. Rev. Lett. 74, 3327 (1995).

[16] M. Cacciari, M. Greco, M. L. Mangano, and A. Petrelli, CERN preprint CERN-TH/95129, hep-ph/9505379 (May 1995).

[17] Talk given by V. Papadimitriou (CDF Coll.), FERMILAB-CONF-94-221-E, contribution to DPF'94 meeting at Albuquerque, NM (August 1994). 
[18] E. Eichten and C. Quigg, Phys. Rev. D49, 5845 (1994), and references therein.

[19] E. Bagan, H. G. Dosch, P. Gosdzinsky, S. Narison, and J.-M. Richard, Z. Phys. C64, 57 (1994).

[20] V. V. Kiselev, A. K. Likhoded, and A. V. Tkabladze, Serpukhov preprint IFVE-94-51, hep-ph/9406339 (April 1994); S. S. Gershtein, V. V. Kiselev, A. K. Likhoded, and A. V. Tkabladze, Serpukhov preprint IFVE-94-81, hep-ph/9504319 (April 1995).

[21] Particle Data Group, Phys. Rev. D50, 1323 (1994).

[22] L. Claveli, Phys. Rev. D26, 1610 (1982); C.-H. Chang and Y.-Q. Chen, Phys. Rev. D46, 3845 (1993); Phys. Lett. B284, 127 (1992); V. V. Kiselev, A. K. Likhoded, and M. V. Shevlyagin, Z. Phys. C63, 77 (1994).

[23] C.-H. Chang and Y.-Q. Chen, Phys. Rev. D48, 4086 (1993); C.-H. Chang, Y.-Q. Chen, G.-P. Han, and H.-T. Jiang, Beijing preprint AS-ITP-94-24, hep-ph/9408242 and revised version (June 1995).

[24] S. R. Slabospitsky, Serpukhov preprint IFVE-94-53, hep-ph/9404346 (April 1994).

[25] A. V. Berezhnoy, A. K. Likhoded, and M. V. Shevlyagin, Serpukhov preprint IFVE94-48, hep-ph/9408284 (March 1994); A. V. Berezhnoy, A. K. Likhoded, and O. P. Yushchenko, Serpukhov preprint IFVE-95-59, hep-ph/9504302 (April 1995) and revised version.

[26] M. Masetti and F. Sartogo, Roma preprint 1099-1995, hep-ph/9503491 (April 1995).

[27] K. Kolodziej, A. Leike, and R. Ruckl, Max Planck Institute preprint, MPI-PhT/95-36, hep-ph/9505298 (May 1995).

[28] K. Cheung, Phys. Rev. Lett. 71, 3413 (1993).

[29] K. Cheung and T. C. Yuan, Phys. Lett. B325, 481 (1994). 
[30] K. Cheung and T. C. Yuan, U. T. Austin - U. C. Davis preprint CPP-95-13/UCD-95-24, hep-ph/9510208 (October 1995).

[31] F. Abe et al. (CDF Coll.), FERMILAB-CONF-95-202-E, paper submitted to LeptonPhoton 95 Conference, Beijing P.R. China, August 1995.

[32] CTEQ Collaboration, J. Botts et al., Phys. Lett. B304, 159 (1993).

[33] G. T. Bodwin, E. Braaten, and G. P. Lepage, Phys. Rev. D51, 1125 (1995).

[34] A. K. Likhoded, S. R. Slabospitsky, M. Mangano, and G. Nardulli, Nucl. Instrum. Methods A333, 209 (1993), and references therein.

[35] Talk given by G. Jackson at the Beyond the Standard Model IV Conference, Tahoe City, CA (December 13-18, 1994).

[36] E. Braaten, K. Cheung, S. Fleming, and T. C. Yuan, Phys. Rev. D51, 4819 (1995).

[37] K. Cheung and T. C. Yuan, Phys. Rev. D50, 3181 (1994). 
Table I: Input parameters to the perturbative QCD fragmentation functions for $n=1$ and $n=2$.

\begin{tabular}{ccc}
\hline \hline & $n=1$ & $n=2$ \\
\hline$m_{b}$ & $4.9 \mathrm{GeV}$ & $4.9 \mathrm{GeV}$ \\
$m_{c}$ & $1.5 \mathrm{GeV}$ & $1.5 \mathrm{GeV}$ \\
$R_{n S}(0)$ & $1.28 \mathrm{GeV}^{3 / 2}$ & $0.99 \mathrm{GeV}^{3 / 2}$ \\
$H_{1}$ & $10 \mathrm{MeV}$ & $14 \mathrm{MeV}^{2}$ \\
$H_{8}^{\prime}(m)$ & $1.3 \mathrm{MeV}[7]$ & $1.8 \mathrm{MeV}[7]$ \\
$\cos \theta_{\text {mix }}$ & 0.999 & 0.957 \\
\hline \hline
\end{tabular}

Table II: The inclusive production cross sections for the $B_{c}$ meson at the Tevatron including the contributions from all the $\mathrm{S}$-wave and $\mathrm{P}$-wave states below the $B D$ threshold as a function of $p_{T}^{\min }\left(B_{c}\right)$. The acceptance cuts are $p_{T}\left(B_{c}\right)>6 \mathrm{GeV}$ and $\left|y\left(B_{c}\right)\right|<1$.

\begin{tabular}{cccc}
\hline \hline$p_{T}^{\min }(\mathrm{GeV})$ & & $\sigma(\mathrm{nb})$ & \\
\hline & $\frac{\mu=\frac{1}{2} \mu_{R}}{2.81}$ & $\frac{\mu=\mu_{R}}{5.43}$ & $\underline{\mu=2 \mu_{R}}$ \\
6 & 0.87 & 1.16 & 6.93 \\
10 & 0.26 & 0.29 & 1.22 \\
15 & 0.098 & 0.097 & 0.26 \\
20 & 0.021 & 0.018 & 0.083 \\
30 & & & 0.014 \\
\hline
\end{tabular}


Table III: The inclusive production cross sections for the $B_{c}$ meson at the LHC including the contributions from all the $\mathrm{S}$-wave and $\mathrm{P}$-wave states below the $B D$ threshold as a function of $p_{T}^{\min }\left(B_{c}\right)$. The acceptance cuts are $p_{T}\left(B_{c}\right)>10 \mathrm{GeV}$ and $\left|y\left(B_{c}\right)\right|<2.5$.

\begin{tabular}{cc}
\hline \hline$p_{T}^{\min }(\mathrm{GeV})$ & $\sigma(\mathrm{nb}), \mu=\mu_{R}$ \\
\hline 10 & 33.8 \\
20 & 4.4 \\
30 & 1.1 \\
40 & 0.41 \\
\hline
\end{tabular}

\section{Figure Captions}

1. The differential cross section $d \sigma / p_{T}$ versus $p_{T}$ of the $(\bar{b} c)$ meson $(H)$ in various spinorbital states with $n=1$ at the Tevatron. The acceptance cuts are $p_{T}(H)>6 \mathrm{GeV}$ and $|y(H)|<1$.

2. Same as Fig.1 for $n=2$.

3. The integrated cross section $\sigma\left(p_{T}>p_{T}^{\min }\right)$ versus the minimum $p_{T}^{\min }$ cut on the $(\bar{b} c)$ meson $(H)$ in various spin-orbital states with $n=1$ at the Tevatron. The acceptance cuts are the same as Fig.1.

4. Same as Fig.3 for $n=2$.

5. The comparison of the differential cross sections $d \sigma / d p_{T}$ at different factorization scales for the $1{ }^{1} S_{0}$ and $1{ }^{3} P_{0}$ states at the Tevatron. The acceptance cuts are the same as Fig.1. 
Fig. 1

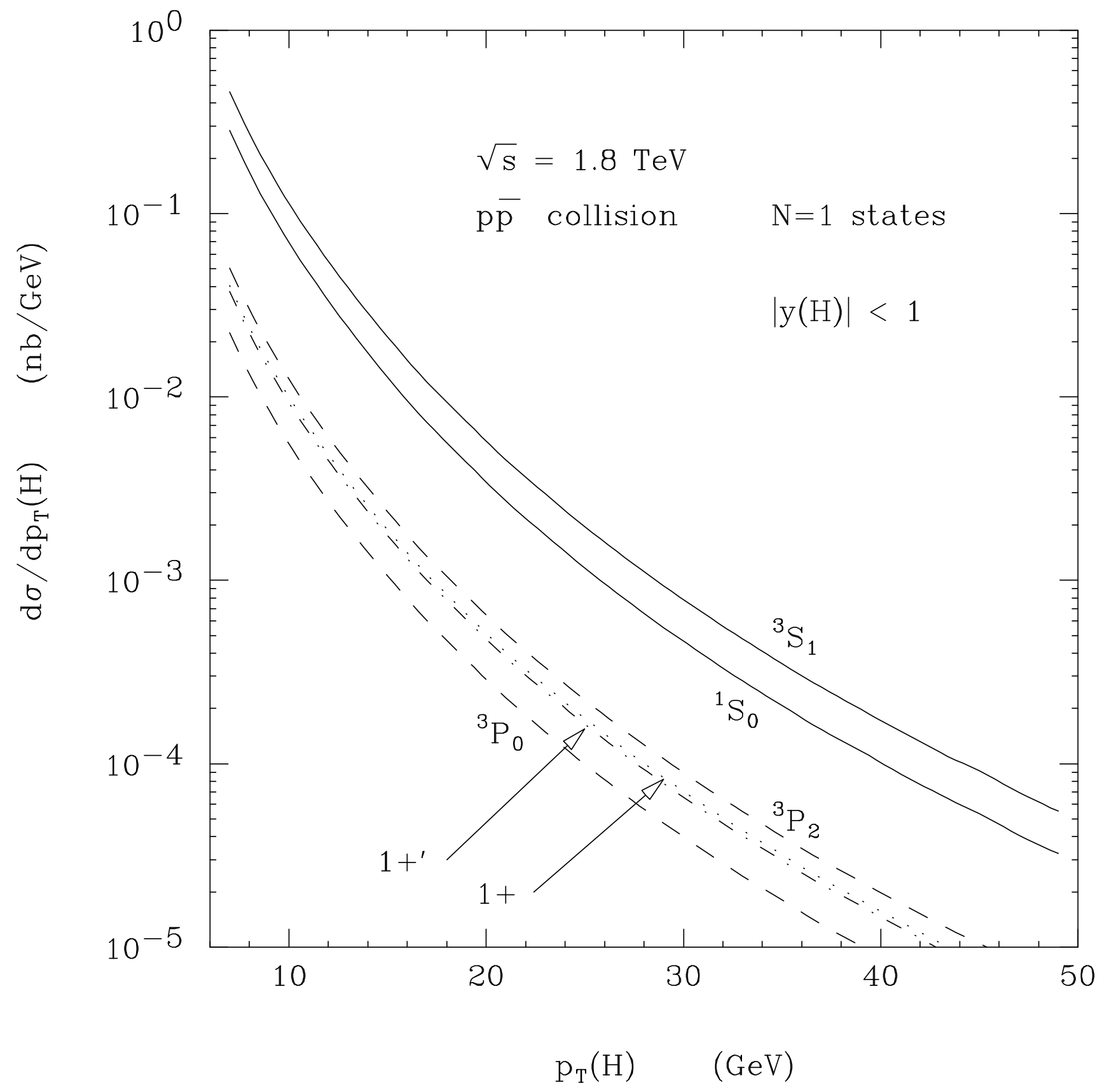


Fig. 2

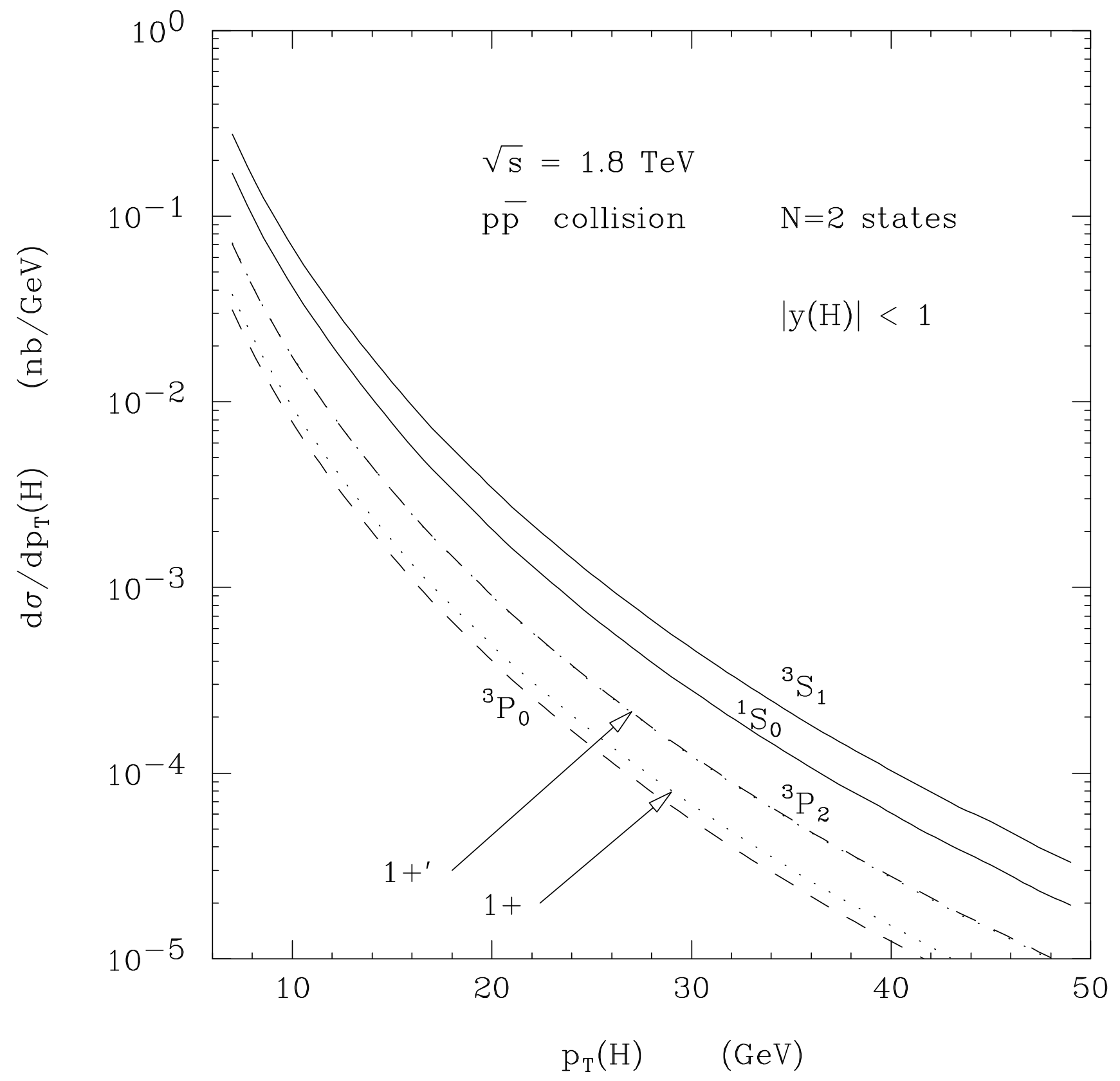


Fig. 3

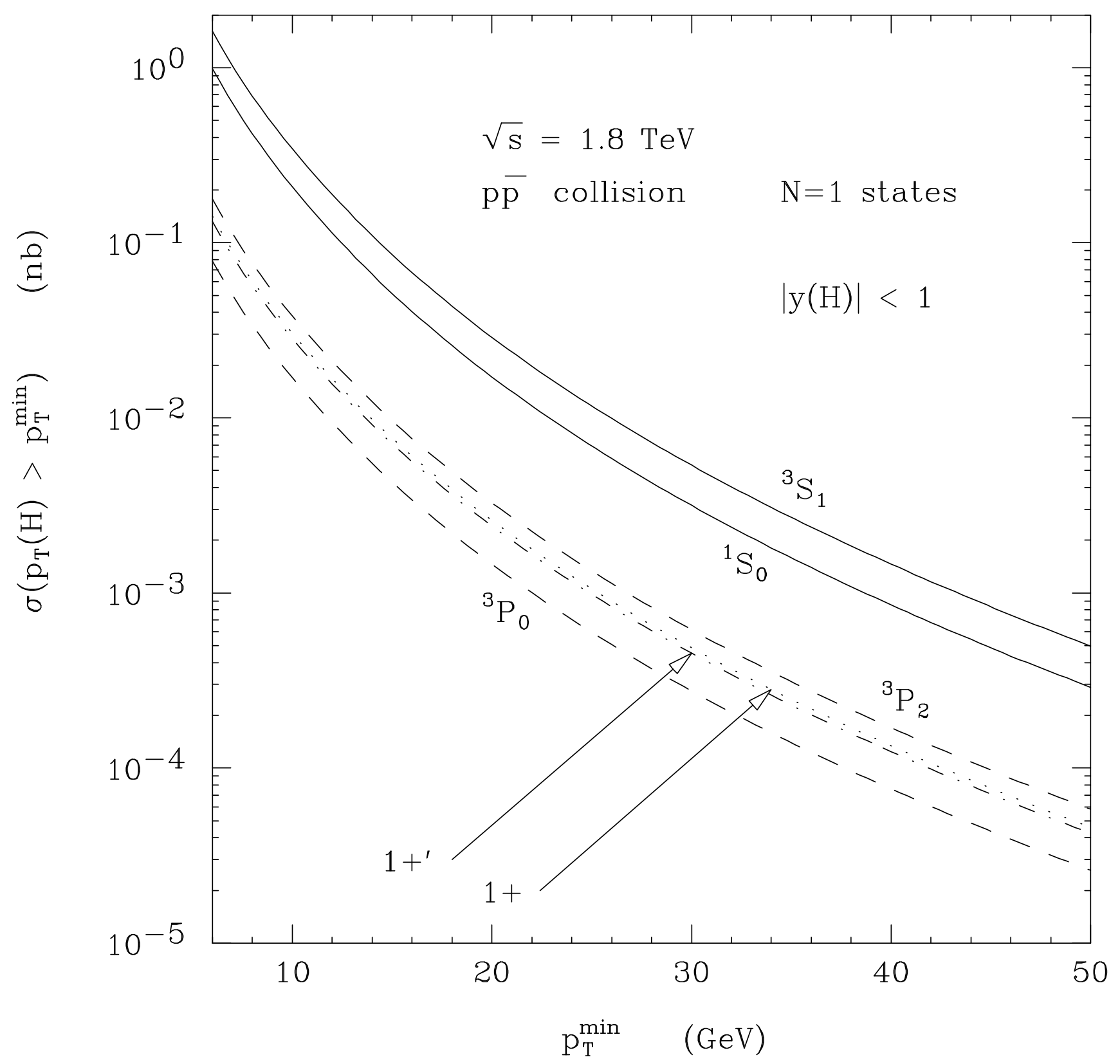


Fig. 4

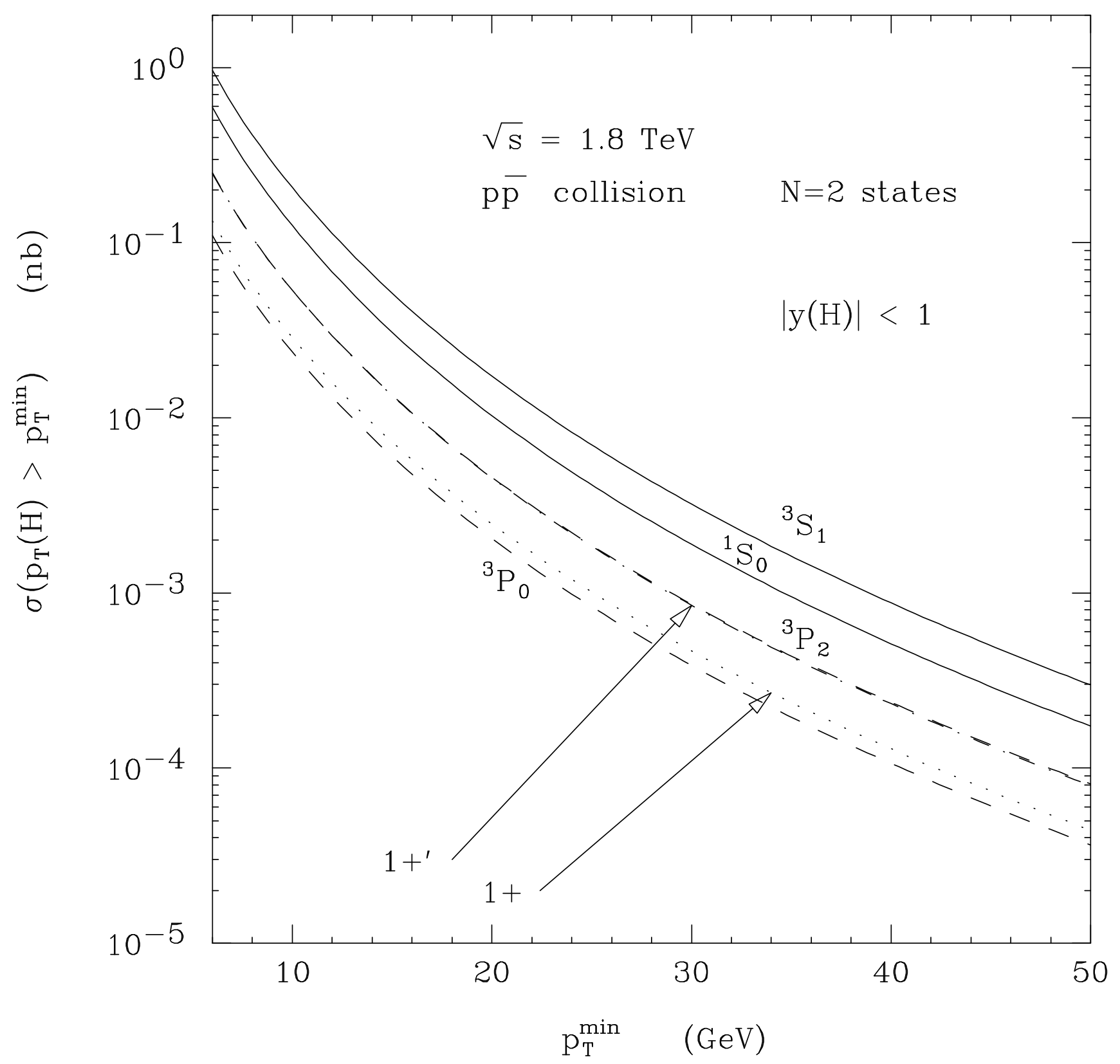


Fig. 5

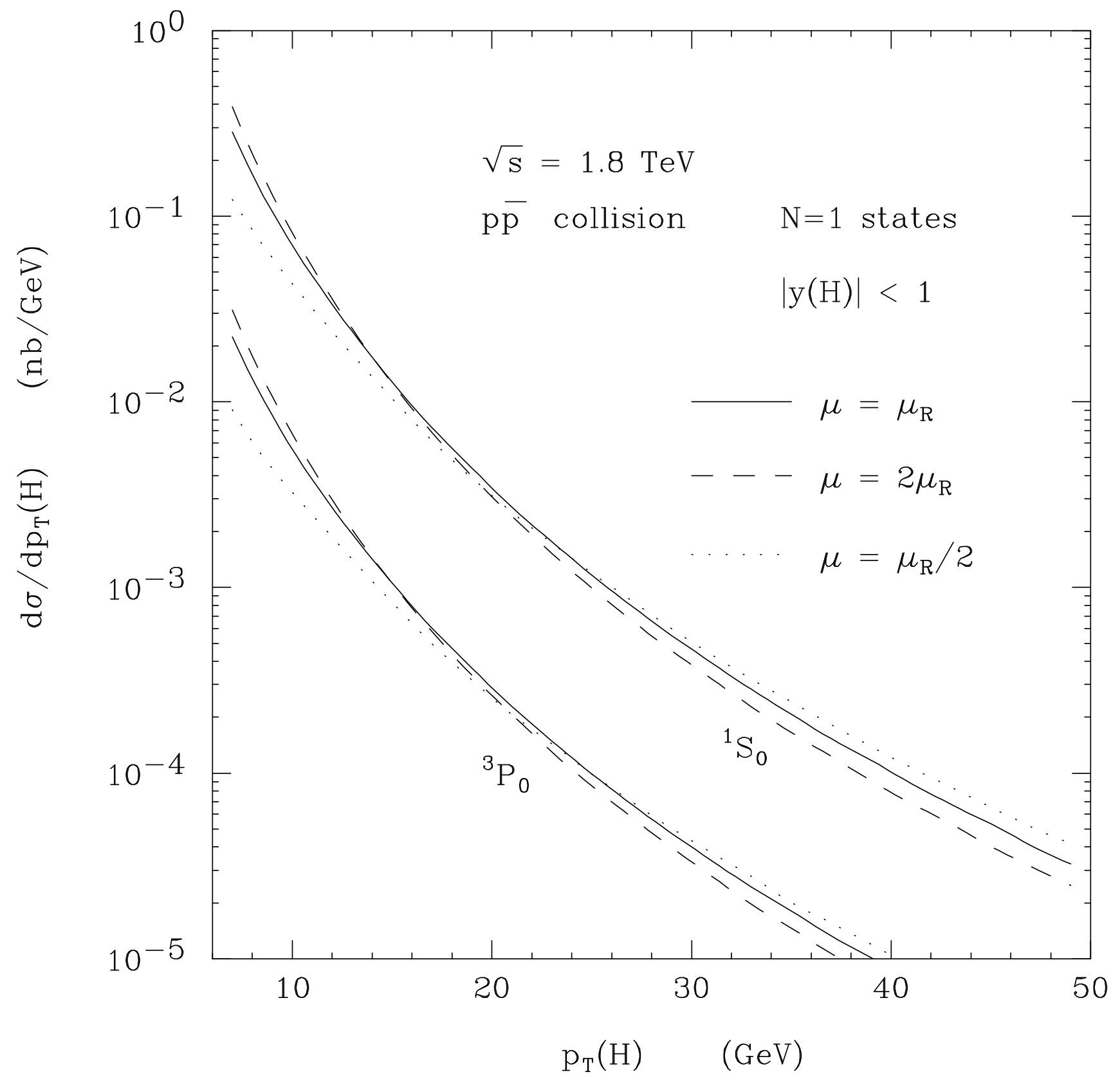

